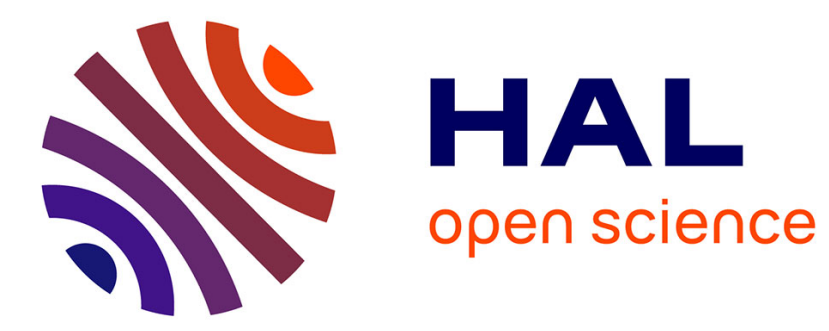

\title{
Monotone continuous multiple priors
}

Alain Chateauneuf, Fabio Macheronni, Massimo Marinacci, Jean-Marc Tallon

\section{To cite this version:}

Alain Chateauneuf, Fabio Macheronni, Massimo Marinacci, Jean-Marc Tallon. Monotone continuous multiple priors. Economic Theory, 2005, 26 (4), pp.973-982. 10.1007/s00199-004-0540-2 . halshs00177057

\section{HAL Id: halshs-00177057 https://shs.hal.science/halshs-00177057}

Submitted on 5 Oct 2007

HAL is a multi-disciplinary open access archive for the deposit and dissemination of scientific research documents, whether they are published or not. The documents may come from teaching and research institutions in France or abroad, or from public or private research centers.
L'archive ouverte pluridisciplinaire HAL, est destinée au dépôt et à la diffusion de documents scientifiques de niveau recherche, publiés ou non, émanant des établissements d'enseignement et de recherche français ou étrangers, des laboratoires publics ou privés. 


\title{
Monotone Continuous Multiple Priors*
}

\author{
Massimo Marinacci Fabio Maccheroni Alain Chateauneuf Jean-Marc Tallon ${ }^{\dagger}$ \\ Submitted: November, 2002 - Revised: June, 2003
}

\begin{abstract}
In a multiple priors model à la Gilboa and Schmeidler (1989), we provide necessary and sufficient behavioral conditions ensuring the countable additivity and non-atomicity of all priors.

Key words: non-expected utility, multiple priors, countable additivity, non-atomicity.

JEL Classification: D81.
\end{abstract}

\section{Introduction}

Decision theorists have often debated whether to use countably or finitely additive probabilities to model decision makers' subjective beliefs. The two most notable advocates of finite additivity were de Finetti and Savage, who argued that countable additivity is a purely technical property devoid of a clear behavioral content and whose assumption prevents the analysis of significant phenomena (see Savage, 1954, and de Finetti 1931, and 1970).

On the other hand, countable additivity is a very convenient property, which leads to many important results in probability theory like, for example, the classic limit laws. As a result, its use is pervasive in mathematical economics and finance. For a decision theorist the problem is, therefore, to understand whether the added analytic power of countable additivity offsets its supposed shaky behavioral underpinning.

An important contribution to this issue was provided by Arrow (1970), who identified the precise behavioral conditions under which subjective beliefs can be represented by a countably additive probability. By building on Villegas (1964), Arrow (1970) obtained a subjective expected utility representation with a countably additive probability by adding the following monotone continuity axiom to a set of standard Savage-type axioms.

Axiom 1 (Monotone Continuity) Given any acts $f \succ g$ in $L$, consequence $x$ in $X$, and sequence of events $\left\{E_{n}\right\}_{n \geq 1}$ in $\Sigma$ with $E_{1} \supseteq E_{2} \supseteq \ldots$ and $\bigcap_{n \geq 1} E_{n}=\emptyset$, there exists $\bar{n} \geq 1$ such that

$$
\left[\begin{array}{ll}
x & \text { if } s \in E_{\bar{n}} \\
f(s) & \text { if } s \notin E_{\bar{n}}
\end{array}\right] \succ g \quad \text { and } \quad f \succ\left[\begin{array}{ll}
x & \text { if } s \in E_{\bar{n}} \\
g(s) & \text { if } s \notin E_{\bar{n}}
\end{array}\right] .
$$

\footnotetext{
*We wish to thank Erio Castagnoli, Larry Epstein, Paolo Ghirardato, Itzhak Gilboa, Luigi Montrucchio, David Schmeidler, Marciano Siniscalchi, an Associate Editor, and an anonymous Referee for helpful discussions. Fabio Maccheroni and Massimo Marinacci gratefully acknowledge the financial support of MIUR (Ministero dell'Istruzione, dell'Università e della Ricerca).

${ }^{\dagger}$ Marinacci is at Dipartimento di Statistica e Matematica Applicata, Università di Torino, Piazza Arbarello 8, 10122 Torino, Italy, and ICER (Torino), massimo.marinacci@unito.it; Maccheroni is at Istituto di Metodi Quantitativi and IGIER, Università Bocconi, Viale Isonzo 25, 20135 Milano, Italy, and ICER, fabio.maccheroni@uni-bocconi.it; Chateauneuf is at CERMSEM, Université de Paris I, 106-112, Bld de l'Hôpital, 75647 Paris, France, chateaun@univ-paris1.fr; Tallon is at CNRS-EUREQua, Université de Paris I, 106-112, Bld de l'Hôpital, 75647 Paris, France, jmtallon@univ-paris1.fr.
} 
With his result, Arrow (1970) showed that monotone continuity is the behavioral condition which underlies the use of countably additive probabilities in subjective expected utility theory. The question is, therefore, whether or not a most useful condition like monotone continuity is also a sensible behavioral property. It is not, however, our purpose to expatiate on this, ultimately subjective, issue. ${ }^{1}$

In contrast, our aim in this paper is to study the implications of monotone continuity for the multiple priors model, a popular generalization of subjective expected utility theory. In this model the decision makers' beliefs are represented by a set $C$ of priors in order to capture the vagueness of beliefs (also called ambiguity), and acts are ranked according to the minimum expected utilities with respect to $C$ (see Gilboa and Schmeidler, 1989) or, more generally, according to a weighted average of the minimum and the maximum expected utilities with respect to $C$. Conventional subjective expected utility theory is the special case in which the set of priors $C$ is a singleton, modelling in this way a situation where there is no vagueness.

Not surprisingly, countable additivity turns out to be a very convenient property in applications of the multiple priors model. For example, the recent applications in economics and finance of Epstein and Wang (1994) and (1995), Billot, Chateauneuf, Gilboa, and Tallon (2000), Delbaen (2000), and Chen and Epstein (2002) critically depend on the countable additivity of the probabilities forming the decision makers' set of priors, and on some compactness properties of such a set.

It is natural to wonder whether such a convenient property has its behavioral counterpart in the monotone continuity of preferences. Theorem 1 below shows that, fortunately, this is indeed the case. In particular, a preference relation having a multiple priors representation is monotone continuous if and only if the set of priors is a relatively weak compact subset of countably additive probabilities.

As well-known, the subjective probability derived in Savage (1954) is convex ranged. This is another convenient property, which recently has been used in a multiple priors setting (see Nehring, 2001, and Amarante, 2002 and 2003). Theorem 2 below extends another classic result of Villegas (1964) by showing that a simple atomlessness property of preferences is a necessary and sufficient condition for the range convexity of all the priors in $C$, while Theorem 3 shows that a more demanding property is needed to obtain the convex-rangedness of the whole set $C$ as a vector measure. Finally, all proofs are relegated to the Appendix.

\section{Set-up}

\subsection{Mathematical Preliminaries}

Throughout the paper, $\Sigma$ is a $\sigma$-algebra of subsets of a space $S$. Subsets of $S$ are understood to be in $\Sigma$ even where not stated explicitly.

We denote by $b a(S, \Sigma)$ and $c a(S, \Sigma)$, respectively, the vector spaces of finitely additive and countably additive bounded real-valued set functions on $\Sigma$; we call charges the elements of ba $(S, \Sigma)$ and measures the elements of $c a(S, \Sigma)$. Clearly, $c a(S, \Sigma)$ is a vector subspace of $b a(S, \Sigma)$. In particular, both $c a(S, \Sigma)$ and $b a(S, \Sigma)$ become Banach spaces when equipped with the variation norm. An element $\mu$ of $c a(S, \Sigma)$ is non-atomic if, for all $A \in \Sigma$ with $\mu(A) \neq 0$, there exists $B \in \Sigma$ such that $B \subseteq A$ and $\mu(A) \neq \mu(B) \neq 0$; non-atomic elements of $c a(S, \Sigma)$ form a closed subspace of $c a(S, \Sigma)$ (see, e.g., Aumann and Shapley 1974 p. 28).

We denote by $B(S, \Sigma)$ the set of all bounded and $\Sigma$-measurable functions $\varphi: S \rightarrow \mathbb{R}$. The vector space $B(S, \Sigma)$ is a Banach space with respect to the supnorm $\|\cdot\|_{s}$. The standard duality between $b a(S, \Sigma)$ and

\footnotetext{
${ }^{1}$ It may be interesting to know Arrow's opinion. In Arrow (1970) he wrote that "the assumption of Monotone Continuity seems, I believe correctly, to be the harmless simplification almost inevitable in the formalization of any real-life problem."
} 
$B(S, \Sigma)$ endows $b a(S, \Sigma)$ and its subsets of a weak* topology. ${ }^{2}$

Finally, $b a^{1}(S, \Sigma)$ and $c a^{1}(S, \Sigma)$ denote, respectively, the sets of probabilities in $b a(S, \Sigma)$ and $c a(S, \Sigma)$; we reserve the letter $P$ for elements of $b a^{1}(S, \Sigma)$ and $c a^{1}(S, \Sigma)$.

\subsection{Decision-theoretic Preliminaries}

States of nature and events are represented by the pair $(S, \Sigma)$, while $X$ is the space of consequences. An act is a map $f: S \rightarrow X$ and it is simple when it is finite valued; $L_{0}$ denotes the set of all simple $\Sigma$-measurable acts. The decision maker has a preference relation $\succsim$ on $L_{0}$, which in turn induces a preference over $X$, obtained in the standard way by identifying consequences with constant acts.

A binary relation $\succsim$ on $L_{0}$ is an $\alpha$-maximin expected utility $(\alpha$-MEU) preference relation if there exists a utility index $u: X \rightarrow \mathbb{R}$, a non-empty set $C \subseteq b a^{1}(S, \Sigma)$ and a constant $\alpha \in[0,1]$ such that $\succsim$ is represented by the preference functional $V: L_{0} \rightarrow \mathbb{R}$ defined by

$$
V(f)=\alpha \inf _{P \in C} \int u(f(s)) d P(s)+(1-\alpha) \sup _{P \in C} \int u(f(s)) d P(s)
$$

for all $f \in L_{0}$. When $C=\{P\}$ is a singleton, $\alpha$-MEU preferences collapse to the Subjective Expected Utility (SEU) case $V(f)=\int u(f(s)) d P(s)$.

We assume that the range $u(X)$ of $u$ is not a nowhere dense subset of $\mathbb{R}$, that is, the interior of the closure $\overline{u(X)}$ is non-empty. This is the case when $u(X)$ is an interval of $\mathbb{R}$; for instance, when $X$ is a convex set and $u$ is non-constant and affine, or when $X$ is a connected topological space and $u$ is non-constant and continuous. This assumption implies that $X$ has to be at least countably infinite.

Notice that, given any set of priors $C \subseteq b a^{1}(S, \Sigma)$, we have

$$
V(f)=\alpha \min _{P \in \overline{c o} w *(C)} \int u(f(s)) d P(s)+(1-\alpha) \max _{P \in \overline{c o} w *(C)} \int u(f(s)) d P(s) .
$$

For this reason the set $C$ itself is often assumed to be convex and weak* closed.

Axiomatic characterizations of this kind of preferences for $\alpha=1$ (MEU) can be found in Gilboa and Schmeidler (1989), Casadesus-Masanell, Klibanoff, and Ozdenoren (2000), and Ghirardato, Maccheroni, Marinacci, and Siniscalchi (2001), while the general case of $\alpha \in[0,1]$ is considered in Ghirardato, Maccheroni, and Marinacci (2002) and Kopylov (2002).

Let $L$ be the set of all acts $f: S \rightarrow X$ that are both preference measurable, i.e., $\{s \in S: f(s) \succsim x\}$ and $\{s \in S: f(s) \succ x\}$ belong to $\Sigma$ for all $x$ in $X$, and preference bounded, i.e., there exist $\underline{x}$ and $\bar{x}$ in $X$ such that $\bar{x} \succsim f(s) \succsim \underline{x}$ for all $s \in S$. Since for all $f \in L$ we have $u \circ f \in B(S, \Sigma)$, the natural extension of the functional $V$ defined by (1) from $L_{0}$ to $L$ allows to extend $\succsim$ from $L_{0}$ to $L$ too; the extensions of $V$ and $\succsim$ to $L$ are still denoted by $V$ and $\succsim$.

\section{Monotone Continuity}

We can now state our main result.

Theorem 1 Let $\succsim$ be an $\alpha$-MEU preference relation on $L$, with a set $C$ of priors. Then, the following conditions are equivalent:

(i) $\succsim$ is monotone continuous.

\footnotetext{
${ }^{2}$ Given a subset $M$ of $b a(S, \Sigma)$, the weak* topology is the weakest topology for which all functionals $\mu \mapsto \int \varphi d \mu$ are continuous, where $\varphi \in B(S, \Sigma)$ and $\mu \in M$.
} 
(ii) $C$ is a relatively weak compact subset of $c a(S, \Sigma)$.

If, in addition, $C$ is weak* closed, then (i) is equivalent to:

(iii) $C$ is a subset of $c a(S, \Sigma)$.

This theorem generalizes the aforementioned results of Arrow and Villegas, who dealt with singleton sets of priors. It is also related to some other results in the literature. Schmeidler (1972) p. 220 noticed that the core of a continuous exact game is a weak sequentially compact subset of $c a^{1}(S, \Sigma)$, while Epstein and Wang (1995) p. 44 showed that the set of priors is a weak sequentially compact subset of $c a(S, \Sigma)$ when the MEU functional $\min _{P \in C} \int u(f) d P$ is continuous at certainty. Finally, Marinacci (2002) showed that $C$ is included in $c a(S, \Sigma)$ whenever $\succsim$ is monotone continuous.

Some more behavioral conditions equivalent to (i) can be found in Theorem A.9, while the EberleinSmulian Theorem (see, e.g. Megginson, 1998 p. 248), Theorems IV.9.1 and IV.9.2 of Dunford and Schwartz (1958), and Lemmas A.4 and A.6 provide several topological conditions equivalent to (ii).

\section{Range Convexity}

By using bets, the preference $\succsim$ on $L$ induces in a well-known way a likelihood ordering $\succsim_{l}$ on the event $\sigma$-algebra $\Sigma$, which takes the form $A \succsim_{l} B$ if and only if

$$
\alpha \inf _{P \in C} P(A)+(1-\alpha) \sup _{P \in C} P(A) \geq \alpha \inf _{P \in C} P(B)+(1-\alpha) \sup _{P \in C} P(B) .
$$

Villegas (1964)'s results imply that for a standard monotone continuous SEU ordering $\succsim$, the single probability measure $P$ that represents $\succsim_{l}$ is non-atomic if and only if $\succsim_{l}$ satisfies the following condition.

Axiom 2 (Downward Atomlessness) If $A \succ_{l} \emptyset$, there exists $B \subseteq A$ such that $A \succ_{l} B \succ_{l} \emptyset$.

In the standard SEU case, in which $C$ is a singleton, downward atomlessness is equivalent to:

Axiom 3 (Upward Atomlessness) If $A \prec_{l} S$, there exists $B \supseteq A$ such that $A \prec_{l} B \prec_{l} S$.

For $\alpha \in(0,1)$, downward and upward atomlessness always coincide; for $\alpha \in\{0,1\}$, some further conditions are needed (see Lemma A.11). The next result shows that downward atomlessness is the appropriate non-atomicity requirement for 0-MEU preferences, upward atomlessness is the appropriate one for 1-MEU preferences, and any one of the two works for $\alpha$-MEU preferences when $\alpha \in(0,1)$.

Theorem 2 Let $\succsim$ be a monotone continuous $\alpha$-MEU preference relation on $L$, with a set $C$ of priors. If $\alpha \neq 1$ ( $\alpha \neq 0$, resp.), the following conditions are equivalent:

(i) $\succsim$ is downward atomless (upward, resp.),

(ii) all priors $P$ in $C$ are non-atomic.

As well-known, a probability measure $P$ is non-atomic if and only if it is convex-ranged, that is, if for each $A \in \Sigma$ and each $\alpha \in[0,1]$, there exists $B \subseteq A$ such that $P(B)=\alpha P(A)$. In other words, $P$ is non-atomic if and only if the set $\{P(B): B \subseteq A$ and $B \in \Sigma\}$ is convex and compact in $\mathbb{R}$ for all $A \in \Sigma$.

Given a set $C$ of probability measures, an extension of this property is to require that for each $A \in \Sigma$ the set

$$
\left\{\{P(B)\}_{P \in C}: B \subseteq A \text { and } B \in \Sigma\right\}
$$


is convex and weak compact in the Banach space $B(C)$ of all real-valued bounded functions on $C$. In this case, we say that $C$ is uniformly convex-ranged. In fact, this implies that for each $A \in \Sigma$ and each $\alpha \in[0,1]$, there exists $B \subseteq A$ such that $P(B)=\alpha P(A)$ for all $P \in C .^{3}$

The next result provides a behavioral characterization of the uniform range convexity of the set of priors $C$ of an $\alpha$-MEU preference. To state it we have to introduce few notions. An act $f \in L$ is unambiguous given $A$ if

$$
\int u(f) d P_{A}=\int u(f) d Q_{A}
$$

for all $P, Q \in C$ such that $P(A)$ and $Q(A)$ are nonzero. Intuitively, an act is unambiguous given $A$ if its evaluation is not affected by the perceived ambiguity when beliefs are updated, one by one, according to Bayes rule given that event $A$ obtained. ${ }^{4}$

A set $A \in \Sigma$ is downward null if $A \sim_{l} \emptyset$, while it is upward null if $A^{c} \sim_{l} S$. Finally, an act $f: S \rightarrow X$ is essentially downward (upward, resp.) constant on $A$ if there exists a downward (upward, resp.) null subset $N$ of $A$ and a consequence $x \in X$ such that $f(s) \sim x$ for all $s \in A-N$.

We are now ready to state the promised characterization.

Theorem 3 Let $\succsim$ be a monotone continuous $\alpha-M E U$ preference relation on $L$, with a set $C$ of priors, and suppose $u(X)$ is an interval. If $\alpha \neq 1(\alpha \neq 0$, resp.), the following conditions are equivalent:

(i) For all non downward (upward, resp.) null events $E \in \Sigma$, there exists an act $f \in L$ both unambiguous given $E$ and non essentially downward (upward, resp.) constant on E;

(ii) The set $C$ is uniformly convex-ranged.

In reading condition (i), it is important to observe that essentially constant acts are unambiguous given any event $E$. Hence, it is key in (i) to require the act not to be essentially constant.

\section{A Proofs and Related Material}

\section{A.1 Compactness}

The following result shows a noteworthy relation existing between compactness in the weak and weak* topologies. It is essentially due to Bartle, Dunford and Schwartz (1955) (see Maccheroni and Marinacci, 2001, for a simple proof).

Lemma 4 Let $C$ be a subset of $c a(S, \Sigma)$. Then, the following statements are equivalent:

(i) $C$ is weak* closed and relatively weak compact.

(ii) $C$ is weak* closed and norm bounded.

(iii) $C$ is weak $k^{*}$ compact.

(iv) $C$ is weak* sequentially compact.

(v) $C$ is weak $k^{*}$ limit point compact.

(vi) $C$ is weak compact.

\footnotetext{
${ }^{3}$ For example, this property plays a key role in Nehring (2001).

${ }^{4}$ See Ghirardato, Maccheroni, and Marinacci (2002) for a discussion, as well as the references therein contained.
} 
Moreover, if $C \subseteq \mathrm{ca}^{1}(S, \Sigma)$ is convex and (vi) holds, then there exists $P_{0} \in C$ such that for all $P \in C$ we have $P(A)=0$ whenever $P_{0}(A)=0$.

Another couple of useful lemmas.

Lemma 5 A subset $C$ of $c a(S, \Sigma)$ is norm bounded if and only if $\sup _{\mu \in C}|\mu(A)|<\infty$ for all $A \in \Sigma$.

We omit the proof, which follows from Theorem IV.9.8 and Lemma III.1.5 of Dunford and Schwartz (1958).

Lemma 6 Let $C$ be a subset of $c a(S, \Sigma)$. The following facts are equivalent:

(i) The set map from $\Gamma: \Sigma \rightarrow \mathbb{R}^{C}$ defined by $\Gamma(A)=\{\mu(A)\}_{\mu \in C}$ takes values in $B(C)$ and it is countably additive in $\left(B(C),\|\cdot\|_{s}\right)$.

(ii) $C$ is bounded and $\sup _{\mu \in C}\left|\mu\left(A_{n}\right)\right| \rightarrow 0$ whenever $A_{n} \downarrow \emptyset$.

(iii) $C$ is relatively weak compact.

Again we omit the proof, which builds on our Lemma 5 and on Theorem IV.9.1 of Dunford and Schwartz (1958).

\section{A.2 Monotone Continuity}

Instead of directly proving Theorem 1, it is convenient to prove the slightly more general Theorem 9 below.

If $f, g \in L$ and $A \in \Sigma$, we set

$$
f A g(s)=\left\{\begin{array}{ll}
f(s) & s \in A \\
g(s) & s \in A^{c}
\end{array} .\right.
$$

Clearly, $f A g \in L$.

Definition 1 A binary relation $\succsim$ on $L$ is:

- Strongly monotone continuous (s.m.c.) if for all acts e, $f, g \in L$ with $f \succ g$, and all sequences of events $\left\{E_{n}\right\}_{n \geq 1} \subseteq \Sigma$ with $E_{n} \downarrow \emptyset$, there exists $\bar{n} \in \mathbb{N}$ such that: e $E_{\bar{n}} f \succ g$ and $f \succ e E_{\bar{n}} g$.

- weakly left monotone continuous (w.l.m.c.) if for all $x, y, z \in X$ with $y \succ z$, and all sequences of events $\left\{E_{n}\right\}_{n \geq 1} \subseteq \Sigma$ with $E_{n} \downarrow \emptyset$, there exists $\bar{n} \in \mathbb{N}$ such that: $x E_{\bar{n}} y \succ z$.

- weakly right monotone continuous (w.r.m.c.) if for all $x, y, z \in X$ with $y \succ z$, and all sequences of events $\left\{E_{n}\right\}_{n \geq 1} \subseteq \Sigma$ with $E_{n} \downarrow \emptyset$, there exists $\bar{n} \in \mathbb{N}$ such that: $y \succ x E_{\bar{n}} z$.

- weakly monotone continuous (w.m.c.) if it is weakly right monotone continuous and weakly left monotone continuous.

Using m.c. to abbreviate "monotone continuous", we have

$$
\begin{aligned}
& \text { w.l.m.c. } \\
& \text { s.m.c. } \rightarrow \text { m.c. } \rightarrow \text { w.m.c. } \\
& \text { w.r.m.c. }
\end{aligned}
$$


Lemma 7 Let $\succsim$ be an $\alpha$-MEU preference relation on $L$, with a set $C$ of priors. If $\alpha \neq 1$ ( $\alpha \neq 0$, resp.) and $\succsim$ is weakly right (left, resp.) monotone continuous, then $C$ is a relatively weak compact subset of ca $(S, \Sigma)$.

Proof. Let $E_{n} \downarrow \emptyset$ and let $y, z \in X$ be such that $y \succ z$ and there exists a sequence $\left\{z_{k}\right\}_{k \geq 1}$ of consequences such that $z_{k} \succ z_{k+1} \succ z$ for all $k \geq 1$, and $\lim _{k \rightarrow \infty} u\left(z_{k}\right)=u(z)$. W.l.o.g., set $u(y)=1$ and $u(z)=0$. By weak right monotone continuity, for all $k \in \mathbb{N}$ there exists $\bar{n} \in \mathbb{N}$ such that $y E_{\bar{n}} z \prec z_{k}$. That is, $\alpha \inf _{P \in C} P\left(E_{\bar{n}}\right)+(1-\alpha) \sup _{P \in C} P\left(E_{\bar{n}}\right)<u\left(z_{k}\right)$. As the sequence $\alpha \inf _{P \in C} P\left(E_{n}\right)+(1-\alpha) \sup _{P \in C} P\left(E_{n}\right)$ is decreasing, this implies

$$
\lim _{n \rightarrow \infty}\left(\alpha \inf _{P \in C} P\left(E_{n}\right)+(1-\alpha) \sup _{P \in C} P\left(E_{n}\right)\right)<u\left(z_{k}\right) .
$$

Passing to the limit for $k \rightarrow \infty$, we get

$$
\lim _{n \rightarrow \infty}\left(\alpha \inf _{P \in C} P\left(E_{n}\right)+(1-\alpha) \sup _{P \in C} P\left(E_{n}\right)\right)=0 .
$$

Since $0 \leq \inf _{P \in C} P\left(E_{n}\right) \leq \alpha \inf _{P \in C} P\left(E_{n}\right)+(1-\alpha) \sup _{P \in C} P\left(E_{n}\right)$, (4) implies $\lim _{n \rightarrow \infty}\left(\inf _{P \in C} P\left(E_{n}\right)\right)=$ 0 . Therefore,

$$
\begin{aligned}
0 & =\lim _{n \rightarrow \infty}\left(\alpha \inf _{P \in C} P\left(E_{n}\right)+(1-\alpha) \sup _{P \in C} P\left(E_{n}\right)\right) \\
& =\alpha \lim _{n \rightarrow \infty} \inf _{P \in C} P\left(E_{n}\right)+(1-\alpha) \lim _{n \rightarrow \infty} \sup _{P \in C} P\left(E_{n}\right) \\
& =(1-\alpha) \lim _{n \rightarrow \infty} \sup _{P \in C} P\left(E_{n}\right),
\end{aligned}
$$

and we can conclude

$$
\lim _{n \rightarrow \infty} \sup _{P \in C} P\left(E_{n}\right)=0 .
$$

Hence, for all $E_{n} \downarrow \emptyset$ and all $Q \in C, 0 \leq Q\left(E_{n}\right) \leq \sup _{P \in C} P\left(E_{n}\right)$ implies $Q\left(E_{n}\right) \downarrow 0$, so that $C \subseteq c a(S, \Sigma)$. Eq. (5) yields relative weak compactness by Lemma 6 . The case in which $\alpha \neq 0$ and $\succsim$ is weakly left monotone continuous is analogous.

Lemma 8 Let $\succsim$ be an $\alpha$-MEU preference relation on $L$, with a set $C$ of priors. If $C$ is a relatively weak compact subset of ca $(S, \Sigma)$, then $\succsim$ is strongly monotone continuous.

Proof. Let $e, f, g \in L$ with $f \succ g$, and $\Sigma \ni E_{n} \downarrow \emptyset$. For all $\varepsilon>0$,

$$
A_{n}=\left\{s \in S:\left|u(f(s))-u\left(e E_{n} f(s)\right)\right|>\varepsilon\right\}=E_{n} \cap\{s \in S:|u(f(s))-u(e(s))|>\varepsilon\} \downarrow \emptyset .
$$

Then, by Lemma $6, \lim _{n}\left(\sup _{P \in C} P\left(A_{n}\right)\right)=0$. Hence, there exists $n_{\varepsilon} \in \mathbb{N}$ such that $P\left(A_{n}\right)<\varepsilon$ for all $n \geq n_{\varepsilon}$ and all $P \in C$. Let $M=\max \left\{\|u \circ e\|_{s},\|u \circ f\|_{s}\right\}$. It holds:

$$
\begin{aligned}
\int\left|u \circ f-u \circ\left(e E_{n} f\right)\right| d P & =\int_{A_{n}}\left|u \circ f-u \circ\left(e E_{n} f\right)\right| d P+\int_{A_{n}^{c}}\left|u \circ f-u \circ\left(e E_{n} f\right)\right| d P \\
& \leq 2 M \varepsilon+\varepsilon
\end{aligned}
$$

for all $n \geq n_{\varepsilon}$ and all $P \in C$. Then $\int u \circ\left(e E_{n} f\right) d P \rightarrow \int u \circ f d P$ uniformly with respect to $P \in C$. Whence

$$
\inf _{P \in C} \int u \circ\left(e E_{n} f\right) d P \rightarrow \inf _{P \in C} \int u \circ f d P \text { and } \sup _{P \in C} \int u \circ\left(e E_{n} f\right) d P \rightarrow \sup _{P \in C} \int u \circ f d P,
$$

and so

$$
V\left(e E_{n} f\right) \rightarrow V(f)>V(g) .
$$

Analogously, $V\left(e E_{n} g\right) \rightarrow V(g)<V(f)$, as desired.

The previous lemmas yield the following result, which in turn implies Theorem 1: 
Theorem 9 Let $\succsim$ be an $\alpha$-MEU preference relation on $L$, with a set $C$ of priors. Then, the following conditions are equivalent:

(i) $\succsim$ is monotone continuous.

(ii) $\succsim$ is weakly monotone continuous.

(iii) $C$ is a relatively weak compact subset of $c a(S, \Sigma)$.

(iv) $\succsim$ is strongly monotone continuous.

If, in addition, $C$ is weak $k^{*}$ closed, then (i) is equivalent to:

(v) $C$ is a subset of $c a(S, \Sigma)$.

The next proposition - which immediately follows from Lemmas 7 and 8 , and from Theorem 9 - illustrates the relations existing between weak left and right monotone continuity.

Proposition 10 Let $\succsim$ be an $\alpha-M E U$ preference relation on $L$. Then,

(i) If $\alpha=0$, $\succsim$ is weakly monotone continuous iff it is weakly right monotone continuous.

(ii) If $\alpha \in(0,1)$, $\succsim$ is weakly right monotone continuous iff it is weakly left monotone continuous.

(iii) If $\alpha=1, \succsim$ is weakly monotone continuous iff it is weakly left monotone continuous.

\section{A.3 Range Convexity}

Proof of Theorem 2. By Lemma 4, there exists $P_{0} \in \overline{c o}^{w^{*}}(C)$ such that $P \ll P_{0}$ for all $P \in \overline{c o} w^{*}(C)$.

Let $\alpha \neq 1$ and let $\succsim_{l}$ be downward atomless. We show that $P_{0}$ is non-atomic. Suppose, per contra, that $A$ is an atom for $P_{0}$. Then $P_{0}(A)>0, A \succ_{l} \emptyset,{ }^{5}$ and for all $B \subseteq A$, either $P_{0}(B)=0$ or $P_{0}(B)=P_{0}(A)$. In the former case, $P(B)=0$ for all $P \in C$, so that $B \sim_{l} \emptyset$; in the latter case, $P_{0}(A-B)=0$, so that $P(A)=P(B)$ for all $P \in C$, and so $B \sim_{l} A$. This is a contradiction, since $\succsim_{l}$ is downward atomless. Therefore, $P_{0}$ is non-atomic. As a consequence any $P \in C$ is non-atomic since $P \ll P_{0}$ (see, e.g., Marinacci 1999, p. 360).

Conversely, let $A \in \Sigma$ be such that $A \succ_{l} \emptyset$. Then $\sup _{P \in C} P(A)>0$, so that $P_{0}(A)>0$. Since $P_{0}$ is nonatomic, there exists a decreasing sequence $B_{n}^{\prime} \downarrow B^{\prime}$ such that $B_{n}^{\prime} \subseteq A$ for all $n \in \mathbb{N}$ and $P_{0}\left(B_{n}^{\prime}\right)=\frac{1}{2^{n}} P_{0}(A)$. Then, the sequence $B_{n}=B_{n}^{\prime}-B^{\prime}$ decreases to $\emptyset$, with $B_{n} \subseteq A$ for all $n \in \mathbb{N}$, and $P_{0}\left(B_{n}\right)=\frac{1}{2^{n}} P_{0}(A)$. Thus $\sup _{P \in C} P\left(B_{n}\right) \downarrow 0$, which implies

$$
\alpha \inf _{P \in C} P\left(B_{n}\right)+(1-\alpha) \sup _{P \in C} P\left(B_{n}\right) \downarrow 0
$$

and

$$
\begin{aligned}
\alpha \inf _{P \in C} P\left(B_{n}\right)+(1-\alpha) \sup _{P \in C} P\left(B_{n}\right) & \geq(1-\alpha) \sup _{P \in C} P\left(B_{n}\right) \\
& =(1-\alpha) \max _{P \in \overline{c 0^{*}}(C)} P\left(B_{n}\right) \geq \frac{1-\alpha}{2^{n}} P_{0}(A)>0 .
\end{aligned}
$$

For $n$ large enough,

$$
\alpha \inf _{P \in C} P(A)+(1-\alpha) \sup _{P \in C} P(A)>\alpha \inf _{P \in C} P\left(B_{n}\right)+(1-\alpha) \sup _{P \in C} P\left(B_{n}\right)>0
$$

${ }^{5} \alpha \inf _{P \in C} P(A)+(1-\alpha) \sup _{P \in C} P(A)=\alpha \min _{P \in \overline{c o} w^{*}(C)} P(A)+(1-\alpha) \max _{P \in \overline{c o}} w^{*}(C) P(A)$ 
that is, $A \succ_{l} B_{n} \succ_{l} \emptyset$.

Let $\alpha \neq 0$, and consider the dual likelihood relation $A \succsim^{l} B$ iff $B^{c} \succsim_{l} A^{c}$. Set $\beta=1-\alpha \in[0,1)$ and notice that $\succsim_{l}$ is upward atomless iff $\succsim^{l}$ is downward atomless, and that

$$
\begin{aligned}
A \succsim^{l} B & \Leftrightarrow \alpha \inf _{P \in C} P\left(A^{c}\right)+(1-\alpha) \sup _{P \in C} P\left(A^{c}\right) \leq \alpha \inf _{P \in C} P\left(B^{c}\right)+(1-\alpha) \sup _{P \in C} P\left(B^{c}\right) \\
& \Leftrightarrow \beta \inf _{P \in C} P(A)+(1-\beta) \sup _{P \in C} P(A) \geq \beta \inf _{P \in C} P(B)+(1-\beta) \sup _{P \in C} P(B) .
\end{aligned}
$$

As a result, if $\succsim_{l}$ is upward atomless, then the argument used in the case $\alpha \neq 1$, when applied to $\succsim^{l}$, shows that $C$ consists of non-atomic measures. Conversely, if $C$ consists of non-atomic measures, the argument used in the case $\alpha \neq 1$, shows that $\succsim^{l}$ is downward atomless and $\succsim_{l}$ is upward atomless.

Lemma 11 Let $\succsim$ be a monotone continuous $\alpha$-MEU preference relation on $L$, with a set $C$ of priors.

(a) If $\alpha \in(0,1)$, then $\succsim_{l}$ is downward atomless iff it is upward atomless.

(b) If $\alpha \in\{0,1\}$, then, downward and upward atomlessness coincide provided that, for any $A \in \Sigma$, we have $A^{c} \sim_{l} \emptyset$ if and only if $A \sim_{l} S$.

Proof. A direct proof of (a), not building on monotone continuity is possible. But, under monotone continuity the result immediately follows from Theorem 2. Next we prove (b).

Suppose $\alpha=1$. We first show that downward atomlessness implies upward atomlessness. Let $\inf _{P \in C} P(A)<$ 1. Then $\inf _{P \in C} P\left(A^{c}\right)>0$. In fact, $\inf _{P \in C} P\left(A^{c}\right)=0$ implies $A^{c} \sim_{l} \emptyset$. Then there exists $B^{c} \subseteq A^{c}$ such

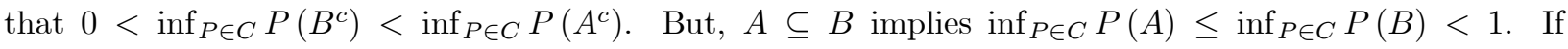
$\inf _{P \in C} P(A)=\inf _{P \in C} P(B)$, then

$$
\begin{aligned}
0 & \leq \inf _{P \in C} P(B-A)=\inf _{P \in C}(P(B)-P(A)) \leq \\
& \leq \inf _{P \in C} P(B)-\inf _{P \in C} P(A)=0,
\end{aligned}
$$

so that $\inf _{P \in C} P\left((B-A)^{c}\right)=1$. In turn, this implies $1-P(B)+P(A)=1$ for all $P \in C$, i.e., $P(A)=P(B)$ for all $P \in C$. Hence, $P\left(A^{c}\right)=P\left(B^{c}\right)$ for all $P \in C$, and so $\inf _{P \in C} P\left(A^{c}\right)=\inf _{P \in C} P\left(B^{c}\right)$, a contradiction.

As to the other implication, let $\inf _{P \in C} P(A)>0$. Then $\inf _{P \in C} P\left(A^{c}\right)<1$, so that there exists $B^{c} \supseteq$ $A^{c}$ such that $\inf _{P \in C} P\left(A^{c}\right)<\inf _{P \in C} P\left(B^{c}\right)<1$. This implies $0<\inf _{P \in C} P(B) \leq \inf _{P \in C} P(A)$. If $\inf _{P \in C} P(A)=\inf _{P \in C} P(B)$, we can proceed as before (exchanging the roles of $B$ and $A$ ) to reach a contradiction.

Suppose $\alpha=0$. Consider the dual likelihood relation $A \succsim^{l} B$ iff $B^{c} \succsim_{l} A^{c}$. Notice that,

1. $A \succsim^{l} B \Leftrightarrow \inf _{P \in C} P(A) \geq \inf _{P \in C} P(B)$.

2. $\succsim_{l}$ is upward atomless iff $\succsim^{l}$ is downward atomless.

3. $\succsim_{l}$ is downward atomless iff $\succsim^{l}$ is upward atomless.

4. $A^{c} \sim^{l} \emptyset$ if and only if $A \sim_{l} S$ if and only if $A^{c} \sim_{l} \emptyset$ if and only if $A \sim^{l} S$.

Hence, the argument used for $\alpha=1$, when applied to $\succsim^{l}$ shows that $\succsim^{l}$ is downward atomless iff $\succsim^{l}$ is upward atomless at $S$, and the same is true for $\succsim_{l}$.

Lemma 12 Let $\succsim$ be a monotone continuous $\alpha$-MEU preference relation on $L$, with a set $C$ of priors, and $P_{0} \in \overline{c o}^{w^{*}}(C)$ be such that $P \ll P_{0}$ for all $P \in \overline{c o}^{w^{*}}(C)$. If $\alpha \neq 1(\alpha \neq 0$, resp.), then $E \in \Sigma$ is downward (upward, resp.) null iff $P_{0}(E)=0$, while $f \in L$ is downward (upward, resp.) essentially constant on $E$ iff $u \circ f$ is $P_{0}$-a.s. constant on $E$. 
Proof. Assume $\alpha \neq 1$; we have

$$
\begin{aligned}
E \sim_{l} \emptyset & \Longleftrightarrow \alpha \inf _{P \in C} P(E)+(1-\alpha) \sup _{P \in C} P(E)=0 \\
& \Longleftrightarrow 0=\sup _{P \in C} P(E)=\max _{P \in \overline{c o}^{*}(C)} P(E) \Longleftrightarrow P_{0}(E)=0 .
\end{aligned}
$$

Next assume $\alpha \neq 0$. Then

$$
\begin{aligned}
& E^{c} \sim_{l} S \Longleftrightarrow \alpha \inf _{P \in C} P\left(E^{c}\right)+(1-\alpha) \sup _{P \in C} P\left(E^{c}\right)=1 \Longleftrightarrow 1=\inf _{P \in C} P\left(E^{c}\right)=\min _{P \in \overline{c o} \omega^{*}(C)} P\left(E^{c}\right) \\
& \Longleftrightarrow P\left(E^{c}\right)=1 \text { for all } P \in \overline{c o}^{w^{*}}(C) \Longleftrightarrow P(E)=0 \text { for all } P \in \overline{c o}^{w^{*}}(C) \Longleftrightarrow P_{0}(E)=0 \text {. }
\end{aligned}
$$

If $\alpha \neq 1(\alpha \neq 0$, resp.), $f: S \rightarrow X$ is downward (upward, resp.) essentially constant on $E$ if there exist a downward (upward, resp.) null subset $N$ of $E$ and a consequence $x \in X$ such that $f(s) \sim x$ for all $s \in E-N$. This happens if and only if there exist a subset $N$ of $E$ such that $P_{0}(N)=0$ and a consequence $x \in X$ such that $u(f(s))=u(x)$ for all $s \in E-N$, which in turn is equivalent to the existence of a subset $N$ of $E$ such that $P_{0}(N)=0$ and $u \circ f$ is constant on $E-N$.

Proof of Theorem 3. We write $\varphi(S) \Subset u(X)$ if there exists a compact interval $H$ such that $\varphi(S) \subseteq H \subseteq$ $u(X)$. It is easy to check that $\{u \circ f: f \in L\}=\{\varphi \in B(S, \Sigma): \varphi(S) \Subset u(X)\}$.

Let $P_{0} \in C$ be such that $P \ll P_{0}$ for all $P \in \overline{c o}^{w^{*}}(C)$. Notice that for a function $\psi$ in $L_{\infty}\left(P_{0}\right)$ the following facts are equivalent:

- $\frac{1}{P(E)} \int_{E} \psi d P=k$ for all $P \in C$ such that $P(E) \neq 0$.

- $\int_{S}\left(\psi 1_{E}+k 1_{E^{c}}\right) d P=k$ for all $P \in C$.

In view of the previous observations, condition (i) becomes:

(ia) for all $E \in \Sigma$ such that $P_{0}(E) \neq 0$, there exist $f \in L$ and $k \in \mathbb{R}$ such that $(u \circ f)_{\mid E}$ is non $P_{0}$-a.s. constant on $E$ and $\frac{1}{P(E)} \int_{E} u \circ f d P=k$ for all $P \in C$ such that $P(E) \neq 0$.

Setting $\varphi=(u \circ f) 1_{E}+k 1_{E^{c}}$, (ia) implies:

(ib) for all $E \in \Sigma$ such that $P_{0}(E) \neq 0$, there exist $\varphi$ in $L_{\infty}\left(P_{0}\right)$ and $k \in \mathbb{R}$ such that $\varphi$ is a.s. equal to $k$ on $E^{c}$ and non a.s. constant on $E$ such that $\int_{S} \varphi d P=k$ for all $P \in C$.

Conversely, if (ib) holds, we can take a representative $\varphi^{\prime}$ of $\varphi$ which is bounded, equal to $k$ on $E^{c}$, and such that $\varphi^{\prime}(S) \Subset u(X){ }^{6}$ Hence, there exists $g \in L$ such that $u \circ g=\varphi^{\prime}$. Clearly, $g$ satisfies (ia), and so (i), (ia), and (ib) are equivalent.

Moreover, (ib) is equivalent to

(ic) for all $E \in \Sigma$ such that $P_{0}(E)>0$, there exists $\varphi$ in $L_{\infty}\left(P_{0}\right)$ which is vanishing off $E$, non-vanishing on $E$, and $\int_{S} \varphi d P=0$ for all $P \in C$.

Finally, observe that $P_{0}(E)=0$ iff $P(F)=0$ for all $P \in C$ and all $F \in \Sigma \cap E$; that is, $\Gamma(F)=0$ for all $F \in \Sigma \cap E$, where $\Gamma: \Sigma \rightarrow B(C)$ is the vector measure defined by $\Gamma(A)=\{P(A)\}_{P \in C}$. By the Knowles Theorem (see Diestel and Uhl, 1977 p. 263), (ic) is then equivalent to (ii).

\footnotetext{
${ }^{6}$ In fact, $u(X)$ has a nonempty interior, and if $\varphi$ and $k$ satisfy (ib), then also $\alpha \varphi+\beta$ and $\alpha k+\beta$ do, for all $\alpha>0$ and all $\beta \in \mathbb{R}$.
} 


\section{References}

[1] C.D. Aliprantis and K.C. Border (1999), Infinite Dimensional Analysis, Springer, New York.

[2] M. Amarante (2002), Ambiguity, measurability and multiple priors, mimeo.

[3] M. Amarante (2003), States, models and unitary equivalence, mimeo.

[4] K. Arrow (1970), Essays in the Theory of Risk-Bearing, North-Holland.

[5] R.J. Aumann and L.S. Shapley (1974), Values of Non-Atomic Games, Princeton University Press, Princeton NJ.

[6] R.G. Bartle, N. Dunford, and J.T. Schwartz (1955), Weak compactness and vector measures, Canad. J. Math 7, 289-305.

[7] A. Billot, A. Chateauneuf, I. Gilboa, and J.-M. Tallon (2000), Sharing beliefs: between agreeing and disagreeing, Econometrica 68, 685-694.

[8] R. Casadesus-Masanell, P. Klibanoff, and E. Ozdenoren (2000), Maxmin expected utility over Savage acts with a set of priors, J. Econ. Theory 92, 35-65.

[9] Z. Chen, and L.G. Epstein (2002), Ambiguity, risk, and asset returns in continuous time, Econometrica 70, 1403-1443.

[10] B. de Finetti (1931), Sul significato soggettivo della probabilità, Fund. Math. 17, 298-329. (English translation in B. de Finetti, Probabilità ed Induzione, CLUEB, Bologna, 1993).

[11] B. de Finetti (1970), Teoria della Probabilità, Einaudi, Turin. (English translation: Theory of Probability, Wiley, New York, 1974).

[12] F. Delbaen (2000). Coherent risk measures in general probability spaces, mimeo.

[13] J. Diestel and J.J. Uhl (1977), Vector Measures, Math. Surveys No. 15. Amer. Math. Soc., Providence RI.

[14] N. Dunford, and J.T. Schwartz (1958), Linear Operators, Part I, Interscience, New York.

[15] L.G. Epstein, and T. Wang (1994), Intertemporal asset pricing under Knightian uncertainty, Econometrica $62,283-322$.

[16] L.G. Epstein, and T. Wang (1995), Uncertainty, risk-neutral measures and security price booms and crashes, J. Econ. Theory 67, 40-82.

[17] P. Ghirardato, F. Maccheroni, M. Marinacci, and M. Siniscalchi (2001), A subjective spin on roulette wheels, Econometrica, forthcoming.

[18] P. Ghirardato, F. Maccheroni, M. Marinacci (2002), Ambiguity from the Differential Viewpoint, ICER WP $17 / 02$.

[19] I. Gilboa and D. Schmeidler (1989), Maxmin expected utility with a non-unique prior, J. Math. Economics 18, 141-153.

[20] G. Knowles (1974), Liapounov vector measures, SIAM J. Control 13, 294-303. 
[21] Kopylov (2002), $\alpha$-Maxmin expected utility, mimeo.

[22] F. Maccheroni and M. Marinacci (2001), A Heine-Borel theorem for ba( $\Sigma)$, Int. Rev. Econ. Bus. 48, 353-362.

[23] M. Marinacci (1999), Upper probabilities and additivity, Sankhya 61, 358-361.

[24] M. Marinacci (2002), Probabilistic sophistication and multiple priors, Econometrica 70, 755-764.

[25] R. E. Megginson (1998), An Introduction to Banach Space Theory, Springer, New York.

[26] K. Nehring (2001), Ambiguity in the context of probabilistic beliefs, mimeo.

[27] L. J. Savage (1954), The Foundations of Statistics, Wiley, New York.

[28] D. Schmeidler (1972), Cores of exact games, J. Math. Anal. Appl. 40, 214-225.

[29] C. Villegas (1964), On qualitative probability $\sigma$-algebras, Ann. Math. Stat. 35, 1787-1796. 